\title{
Causality in Solving Economic Problems
}

\author{
A. Emanuel Robinson', Steven A. Sloman'2, York Hagmayer', \\ and Christopher K. Hertzog ${ }^{4}$
}

\begin{abstract}
:
The role of causal beliefs in people's decisions when faced with economic problems was investigated. Two experiments are reported that vary the causal structure in prisoner's dilemma-like economic situations. We measured willingness to cooperate or defect and collected justifications and think-aloud protocols to examine the strategies that people used to perform the tasks. We found: (i) Individuals who assumed a direct causal influence of their own action upon their competitor's action tended to be more cooperative in competitive situations. (ii) A variety of different strategies was used to perform these tasks. (iii) Strategies indicative of a direct causal influence led to more cooperation. (iv) Temporal cues were not enough for participants to infer a particular causal relation. It is concluded that people are sensitive to causal structure in these situations, a result consistent with a causal model theory of choice (Sloman \& Hagmayer, 2006).
\end{abstract}

\section{Keywords:}

cognitive processes, decision making, behavioral economics, problem solving, causal reasoning

\footnotetext{
This study was part of the lead author's dissertation while at the Georgia Institute of Technology. The lead author is now a Senior Research Scientist at Westat, Inc. and can be contacted at emanuelrobinson@westat .com with correspondence concerning this article. The authors gratefully acknowledge the helpful comments and suggestions of Wendy Rogers, Dan Fisk, Richard Catrambone, Robert Goldstone, and two anonymous reviewers. Also, we thank Amanda Weinberg, Bethany Geist, Shannon Langston, and the rest of the Hertzog Adult Cognition Lab for help with data collection and coding. This research was supported in part by NSF Award 0518147 to Steven Sloman and by an $\mathrm{NIH}$ award to Christopher Hertzog from the National Institute on Aging (R37 AG14138).

'Westat, Inc.; ${ }^{2}$ Brown University; ${ }^{3}$ University of Goettingen; ${ }^{4}$ Georgia Institute of Technology
}

The Journal of Problem Solving • volume 3, no. 1 (Fall 2010) 


\section{Introduction}

The literatures on problem solving and causal reasoning have made remarkably little contact. This is surprising because so many real problems concern causal systems (e.g., broken machinery, attempts to change behavior, medical problems, economic problems). As a first step to bridging this gap, we offer an analysis of people's sensitivity to causal structure when solving a particular type of problem. We chose a type that is abstract and yet is similar to others that are amenable to causal analysis, namely, problems involving choosing among options (Hagmayer \& Sloman, 2009). We focus on paper-and-pencil problems that resemble a two-player prisoner's dilemma.

Prisoner's dilemma games have traditionally been analyzed using game-theoretic utility theory (Luce \& Raiffa, 1957; von Neumann \& Morgenstern, 1953). The standard one-shot, non-iterative prisoner's dilemma is a dilemma in the sense that the dominant options for both players lead to a suboptimal state. A dominant option for a player is one whose value for that player is always equal to or greater than the value of any other option. If both players apply the rational principle of dominance under the assumption that the opponent will also behave rationally (Davis, 1985; Luce \& Raiffa, 1957), they will both defect. If instead they both cooperate, then they attain a better result (e.g., a less severe sentence). Table 1 shows the payoff for the player (bold number on the left side in each cell) and the opponent (number on the right in each cell) in a currency buying dilemma (an example stimulus used in Experiment 1).

Table 1. Example of a Prisoner's Dilemma Payoff Table

\begin{tabular}{|l|l|l|}
\cline { 2 - 3 } \multicolumn{1}{c|}{} & Your competitor buys dollars $(\$)$ & Your competitor buys euros $(€)$ \\
\hline You buy dollars $(\$)$ & $\mathbf{\$ 1}$ billion/\$1 billion & $\mathbf{\$ 5 0}$ million $/ \$ 1.2$ billion \\
\hline You buy euros $(€)$ & $\mathbf{\$ 1 . 2}$ billion/\$50 million & $\mathbf{\$ 1 0 0}$ million $/ \$ 100$ million \\
\hline
\end{tabular}

One difference between our problems and traditional prisoner's dilemmas is that ours include a statement of the probability of the opponent's action. This yields an expected value for the player that favors one option while the other option remains dominant.

People tend to cooperate more than rational models would predict in two-player games like prisoner's dilemma (Camerer, 1997) even when performance has real financial consequences (Shafir \& Tversky, 1992). These findings clearly indicate that people not only focus on rational principles when dealing with the dilemma. They also seem to treat it as a problem, which requires taking into account other aspects. There are many possible reasons why people would choose to cooperate in their approach to the problem (for an extensive list, see Markoczy, 2004). Cooperation could result from (i) moral principle; (ii) a desire to appear cooperative; (iii) treating actions as diagnostic of personality

- volume 3, no. 1 (Fall 2010) 
characteristics along with a desire to think of oneself as cooperative or as possessing traits that promote cooperation; (iv) backward causal reasoning manifest as a belief that cooperation will give one positive personality characteristics; or (v) the belief that one's choice will causally influence the opponent. This list is not exhaustive, but these are the possible motives most relevant to the present experiments. The current experiments attempt to distinguish the psychological plausibility of the various hypotheses using problems based on the prisoner's dilemma in economic contexts. In particular, we focus on the possibility that people impose causal structure on the situation.

Traditional theoretical approaches to these types of problems neglect players'beliefs about the causal structure of the problem and the context in which it is embedded (Nozick, 1969). Players may have causal beliefs about how the situation and their own choices will affect the other player's strategy. They may believe, for instance, that people are more likely to cooperate in a scenario concerning nuclear war than one concerning terrorism. They also may have various, potentially non-veridical beliefs about whether their choices affect the other player's choices. For example, a player might believe that his or her action will influence the opponent to make a similar choice in a prisoner's dilemma. This belief could reflect magical thinking or an illusion of control (Langer, 1975; Langer \& Roth, 1975). If people think they have influence over their partner's choice, then they will cooperate. Alternatively, players may believe that a cooperative action creates a more cooperative world. In the context of such a belief, cooperation is a reasonable strategy as it could maximize expected utility (the greater probability that the opponent cooperates if you do and that the opponent defects if you do means that cooperation is more likely to lead to more benefit).In short, if people are sensitive to causal structure when playing two-player games, then in a prisoner's dilemma-like situation, participants should be more likely to cooperate if their choice has a direct causal influence on the opponent's decision (Hagmayer \& Sloman, 2005, 2009; Nozick, 1993; Sloman, 2005; Sloman \& Hagmayer, 2006).

\section{Temporal Cues to Decision Strategies}

Instructions about timing can help distinguish use of different strategies in a problem based on a single-trial prisoner's dilemma (Morris, Sim, \& Girotto, 1998). Morris et al. found that a delay before the opponent's subsequent decision increased the likelihood of cooperation presumably because people felt they had more control in the presence of a delay. A comparable effect of timing without observability has been observed multiple times with other coordination games (reviewed by Weber, Camerer, \& Knez, 2004). This suggests that the illusion of control—the false belief that one's actions are causal—influences at least some responses to prisoner's dilemma-type problems. By contrast, simultaneous decisions eliminate this possibility. The present study attempted to use temporal instructions to differentiate patterns of responses for problems that varied in their causal structure. 


\section{Outline of Experiments}

The main goal of the two experiments reported here was to better understand how causal reasoning affects strategies brought to bear in prisoner's dilemma-type problems and ensuing choices. In both experiments, one-shot prisoner's dilemma-type problems were presented within an economic context. Experiment 1 manipulated the causal structure of the problem and the timing of opponents' responses across multiple scenarios to measure the influence of causal information. In addition, to learn about how participants understood and justified their strategies, we queried them about their strategies and reasons for choices. Experiment 2 collected qualitative strategy reports by means of think-aloud protocols while also varying causal structure. Performance in both experiments was tested using economic/financial scenarios that did not promote a moral imperative to cooperate. We also used multiple one-shot problems instead of single, iterative problems to avoid longer-term strategizing (e.g., gaining reputation) and increase experimental control (cf. Colman, 2003; Goeree \& Holt, 2001).

\section{Experiments}

\section{Experiment 1}

Participants were presented with prisoner's dilemma-like problems in the context of hypothetical scenarios. As a first factor participants' assumptions about the underlying causal structure were manipulated. Two types of causal structure were used, a CommonCause structure in which both participants chose based on the same scenario and payoff matrix and did not know each other's choices, and a Direct-Cause structure in which the participant knew that the opponent would see the participant's choice prior to making his or her own. A third condition (No-Model) did not specify any causal model. We expect that people will believe their choice influences their opponent's choice only under Direct-Cause instructions. If people expect their opponents to defect, then they too should defect, in this case in order to minimize loss. But if your action determines your opponent's action, then your opponent is almost guaranteed to defect as well in this case, thus capping your gain at a relatively low value. So the most sensible thing to do is to cooperate and hope that your opponent responds to your cooperation in kind, by also cooperating. This could be a microcosm of the sort of causal reasoning that makes cooperation so common in the literature. Therefore, we predict that participants will choose the non-dominant option (i.e., cooperation), which maximizes expected value, more under Direct-Cause than under No-Model or Common-Cause instructions. Therefore, we also predict that under a Direct-Cause assumption people will be more likely to use strategies related to expected value and under Common-Cause strategies related to dominance.

- volume 3, no. 1 (Fall 2010) 
As a second factor, temporal information was manipulated. Participants were told that opponents' decisions were made either simultaneously with their own or subsequently. Game theory is usually interpreted to imply that the dominant action will be chosen whenever the opponent's decision is unknown, regardless of the temporal sequence (e.g., Dixit \& Skeath, 1999). However, the illusion of control suggests that individuals should choose the dominant option when choices are simultaneous but be more likely to cooperate when their move precedes the opponent's (in hopes that it will induce the opponent to cooperate; Morris et al., 1998).

We expected an interaction of both factors. Given a Common-Cause model participants should tend to prefer the dominant option regardless of temporal delay, because both simultaneous and delayed choices by the competitor conform to the assumed model. Given no causal information, we expected participants to consider the temporal relations. Because of a general tendency to assume causal influence (Sloman,2005), we expect that people will assume a direct causal relation if the opponent chooses subsequently even when no explicit causal information is given. In contrast, people should tend to assume no direct causal impact if choices are made simultaneously. If a Direct-Cause model is assumed, we expect higher levels of cooperation than if a Common-Cause model is assumed. The Direct-Cause model implies that the opponent's choice has to follow the player's choice. Therefore, temporal assumptions cannot be manipulated in this case without creating contradictions.

\section{Experiment 1 Method}

Participants. Participants were undergraduates from the Georgia Institute of Technology and were given extra course credit for participating. Participants were randomly assigned to two experimental between-subjects conditions (Simultaneous, Sequential). The session lasted approximately one hour.The Sequential group consisted of 57 participants and the Simultaneous group included 59. Five participants (two in the Sequential condition and three in the Simultaneous condition) were excluded because they disregarded instructions or gave facetious responses (i.e., jokes about the scenario topics).

Materials. Participants were presented with six problems, each in the context of a different economic/financial scenario. For example, participants were asked to imagine working for the Bank of Japan and had to decide whether to buy dollars or euros (see Appendix A for the complete scenario). Each scenario included information about the situation and the opponent and asked for a decision based on that information as if they were in the situation. The scenarios were based on one-shot non-iterative prisoner's dilemmas. Participants only made one decision, and there was no reciprocal decision made by the imaginary opponent. A table was presented with payoffs for the participant and the competitor given all possible combinations of choices. Unlike a true prisoner's dilemma, as part of the scenario participants were told that the opponent's decision matched 
their decision $90 \%$ of the time in past situations for that scenario. This information kept the probabilities the same for all problems, allowing only the causal structure and temporal information to vary, while also contrasting dominance versus expected value. This information was constant across both conditions and for all problems, which makes an effect even harder to detect but was necessary to make sure participants were assuming the same baseline probabilities. The participants were then asked to choose one of two options. They were also asked to give a confidence rating about their decision after each answer, ranging from 0 to 100 (with 100 being completely confident).

Design. There were three levels of causal structure (Direct-Cause model, CommonCause model, and No-Model) and two levels of temporal cues (Simultaneous and Sequential) combined in an incomplete block design because Direct-Cause models were not possible in the Simultaneous condition. Causal structure was manipulated withinsubjects, and temporal cues were between-subjects. All participants were given a set of problems that included a Direct-Cause model relation, a Common-Cause model relation, and a No-Model causal relation. For example, the statement of a Direct-Cause in a bidding scenario was:"In the past, you chose the bid level and your competitor waited for you to announce your choice, then made his/her decision based upon yours."This sentence denotes a direct causal relation between the participant's and the opponent's bid levels. A Common-Cause situation referred instead to environmental factors:"In the past, you chose whether or not to buy dollars (\$) or euros $(€)$ and your competitor had to make his/her decision independently. You both independently based your choice on economic data." The No-Model information format was the same except no relation was mentioned (the participant was free to assume the causal structure or to assume no relationship at all). There were two scenarios of each type with different contents. The presentation order of these blocks was fully counter-balanced.

Temporal cues were only manipulated in Common-Cause and No-Model items. Direct-Cause items have an inherent delay, so participants could not reasonably assume a direct cause if told that their decisions were made simultaneously with their opponent's decisions. In other conditions, the Simultaneous group was told that the opponent made his or her decision at the same time as the participant. The Sequential group was told that the opponent made his or her decision following the participant's decision.

Procedure. Participants were randomly assigned to one of two temporal groups (Simultaneous or Sequential) and presented with two Common-Cause, two Direct-Cause, and two No-Model problems. Direct-Cause items were the same for both temporal groups. The order of all pairs of problems was counter-balanced. Participants were tested in groups of up to eight individuals. Materials were presented on paper. Experimenters answered any clarification questions before beginning. After reading and providing an answer, participants were asked to write justifications for their responses immediately after making a choice for each problem. Finally, after completing all of the problems, participants were 
given an open-ended question asking them to identify what was varied between problems (excluding context and numbers in tables).

\section{Experiment 1 Results}

Choice proportions did not significantly differ within a block for Common-Cause and DirectCause items though one of the No-Model problems resulted in slightly different choice proportions than the other. Consequently, the problems of each type were combined for analyses unless stated otherwise (aggregating two Direct-Cause, two Common-Cause, two No-Model problems).

Temporal Cues. The timing of choice for the participant and an imaginary opponent was either sequential (opponent follows) or simultaneous though this was only varied in the Common-Cause and No-Model conditions. First, collapsing across all orders of presentation within groups, we analyzed Common-Cause items and No-Model items with a one-way ANOVA to compare Sequential versus Simultaneous. There was no significant difference, $F<1$ for Common-Cause items. Individuals did not show an effect of timing. This finding indicates that causal information would override inferences drawn from temporal cues. Similarly, No-Model items did not show a significant difference between simultaneous or sequential temporal cues, $F(1,108)=2.87, p>0.05$. This was not predicted. Similar to the Common-Cause items, when individuals were not given any causal information, they were equally likely to choose dominant and non-dominant options regardless of the temporal cues provided. The lack of difference between Simultaneous and Sequential information in No-Model items suggests that temporal information was not used to infer a causal relation. Finally, items were analyzed based on presentation order, and there were no order effects (all $t$ 's $<1$ ).

Causal Models. The main prediction is that participants would choose the non-dominant option more under Direct-Cause than under No-Model or Common-Cause instructions. As predicted, the mean proportion choosing the dominant option under Direct-Cause instructions was 0.51 , lower than under Common-Cause or No-Model instructions $(M=$ 0.64 and $M=0.66$, respectively).Temporal condition and causal model information did not interact $(F<1)$. Therefore, we collapsed across temporal order groups and evaluated the effect of causal model type (Direct-Cause, Common-Cause, No-Model) with a repeatedmeasures ANOVA. The effect was significant, $F(2,218)=7.52, p<0.01, M S E=0.441$.

A possible consequence of the manipulation within participants was that individuals receiving Direct-Cause instructions first would continue to assume a Direct-Cause relation for No-Model items. Consequently, individuals were separated by whether they received a Direct-Cause item first or not.The mean level of choosing the dominant option for individuals on No-Model items when Direct-Cause items were presented first was $M$ $=0.56(S E=0.08)$ compared to $M=0.71(S E=0.04)$ when Direct-Cause items were not presented first. This difference was significant, $t(108)=-2.12, p<0.05$. When individuals 
were first presented with Direct-Cause information, they tended to continue cooperating even in the absence of a Direct-Cause in subsequent problems.

Assumptions about Causal Models. We expected participants to envision specific causal models. To find out if they did we coded their justifications for their responses according to the causal model they indicated. We classified their written responses as either indicating a Direct-Cause assumption (e.g., indication of an attempt to influence the opponent, which could include wording like "makes," "causes," etc.), a Common-Cause assumption (indications of an extraneous influence, such as the economy, on both the participant and the opponent), or as not providing enough information. This causal model coding was done separately and independently by two raters. In the case of disagreements, consensus was reached after discussion.

The easiest cases to interpret occur when the participant's causal model (as indicated by his or her response) aligns with the causal model instructions for that particular item. As expected, manipulating the causal model assumption made a large difference. For the two Direct-Cause items, the mean proportion choosing a dominant option when using a Direct-Cause model were $M=0.32(S E=0.10)$. For the Common-Cause items, the mean proportion choosing the dominant option for participants who assumed a Common-Cause model were $M=0.67(S E=0.12)$. These differences clearly show a stronger shift in levels of cooperation when we restricted analysis to cases in which participants were aware of causal models.

There was also a striking difference in the No-Model condition for people who inferred a Direct-Cause versus a Common-Cause model. Items were not pooled for this analysis. Those who assumed a Common-Cause model were far more likely to choose the dominant option $\left(M_{1}=0.94, S E_{1}=0.03, M_{2}=0.82, S E_{2}=0.005\right)$ than the participants assuming a direct causal influence $\left(M_{1}=0.12, S E_{1}=0.08, M_{2}=0.04, S E_{2}=0.04\right)$. Independent samples $t$-tests showed a significant difference for both scenarios, $t_{1}(85)=-11.96, p<0.001$ and $t_{2}(85)=-9.63, p<0.001$. Someone assuming a Direct-Cause model, even without being given any causal information, is much more likely to cooperate than someone assuming a third Common-Cause influence.

Qualitative Strategy Coding. This coding was done separately and independently from the causal model coding. Because both problems in a model set were constructed to be very similar and had almost identical means and strategy distributions, one of each type (Direct-Cause, Common-Cause, and No-Model) was selected for the analyses in this section. It would not be possible to collapse strategy choices across sets of problems given the qualitative nature of the responses. ${ }^{1}$ Although there were quite a few strategy categories (see Figure 1), the overwhelming majority of responses could be categorized as either Maximizing Expected Value (MEV) - participant chooses the outcome that would have the highest payoff that also takes into account the probability of success, or Dominance/ Payoff (DOP)—participant chooses the option that has the highest payoff or dominates, 
regardless of probability of success. Only these two strategies were chosen by a substantial number of participants ( $>10 \%$ each). Descriptions of the other strategies, including the Stackelberg heuristic (Colman, 2003) and risk aversion, can be found in Appendix B.

Figure 1 also breaks strategies down via the different causal model assumptions. In line with our predictions, participants assuming a Direct-Cause were more likely to pursue an MEV strategy. Participants used an MEV strategy for $35.1 \%$ of the problems, compared to using a DOP strategy on $27.0 \%$ of the problems. In contrast, when assuming a Common-Cause model, $47.7 \%$ of participants preferred the DOP strategy over $28.8 \%$ who maximized expected value.Thus, strategy preferences flipped depending on causal model assumptions.

Figure 1. Percentages of Each Coded Strategy in Exp.1

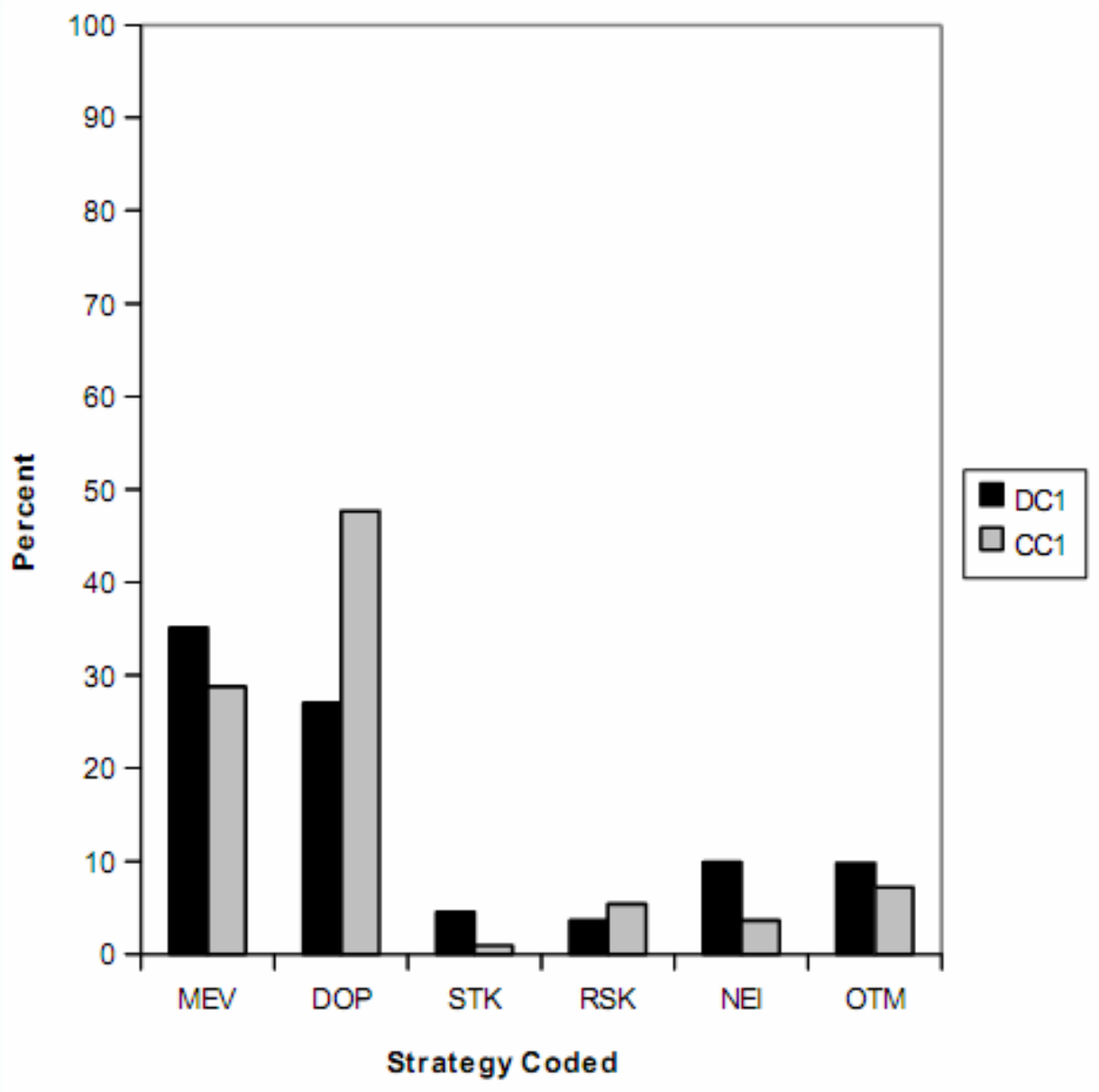

Note: $\mathrm{MEV}=$ Maximizing Expected Value, DOP = Dominance/Payoff, $\mathrm{STK}=$ Stackelberg Heuristic, RSK $=$ Risk Aversion, $\mathrm{NEI}=$ Not Enough Information, $\mathrm{OTM}=$ Other Method. DC1 = Direct-Cause Item 1, CC1 = CommonCause Item 1. 
As expected, strategy choices had a large impact on participants' choices. Given a Direct-Cause scenario the dominant option was much more likely to be chosen by people who looked for the option that yielded the better outcome regardless of the opponent's actions (DOP, mean probability of $0.90, S E=0.06$ ) than by participants who pursued a maximizing expected value strategy $(M=0.10, S E=0.05, t(67)=-10.72, p<0.05)$. The same pattern emerges for the Common-Cause scenario. Of those using the DOP strategy, a much higher proportion chose the dominant option $(M=0.86, S E=0.04)$ than for those individuals who used an MEV strategy $(M=0.19, S E=0.07, t(83)=-9.63, p<0.05)$.

Final Question. A brief,open-ended questionnaire administered after task completion was used to assess whether individuals could identify the causal manipulation between problems. Only $36 \%$ of all participants mentioned "dependence," "affecting opponent," etc. Taken at face value, this indicates individuals need not be consciously aware of the causal information to adjust their choices in this type of task. There has been evidence that during verbal reports individuals are not always able to report higher-order mental processes (e.g., Nisbett \& Wilson, 1977). Also, a plethora of evidence from perception, language, judgment, and reasoning research shows that causal relations structure people's knowledge and thought automatically and effortlessly even when people are not aware of it (for a review, see Sloman, 2005). Experiment 2 will address the issue of what information and strategies people are aware of while making a decision in this context.

Discussion. Overall, we were less successful than we hoped to be at manipulating participants' causal models with temporal cues. There was no effect of the temporal sequences of choices in the No-Model condition. Thus, the present results failed to replicate those of Morris et al. (1998).

By contrast, we found a clear effect of causal model assumptions throughout the experiment. When we inferred causal models from participants'justification, they turned out to be highly predictive of responses. Participants were sensitive to causal model changes across problems. More individuals cooperated on Direct-Cause problems than on other problems. Furthermore, the type of model a participant assumed when given no causal information led to different choices, with Direct-Cause models leading to greater cooperation.

Finally, strategy coding showed individuals shifted strategies based on causal instructions; they looked for the dominant option more often when there was a Common-Cause, but for the option maximizing the expected values when they assumed a direct causal impact. These strategies in turn determined participants' decisions in the end.

\section{Experiment 2}

The main purpose of Experiment 2 was to collect qualitative strategy information in oneshot prisoner's dilemma-type problems by utilizing strategy reports and think-aloud verbal protocols. This allowed us to investigate whether participants are aware of using varied

- volume 3, no. 1 (Fall 2010) 
strategies and whether this has implications for decision-making theories that incorporate models of other players into predictions about a given player in a game situation (e.g., Colman \& Bacharach, 1997; Hedden \& Zhang, 2002; Stahl \& Wilson, 1995). We know of no other investigation using process tracing or think-aloud protocols with this type of task.

In the first experiment participants' strategies and assumptions had to be reconstructed from their justification s for choice. Hence, these answers reflect participants' post hoc self-assessments of the underlying causal model (but not necessarily the strategies used). To gain more insight into the actual decision-making process, we ran a second experiment asking participants to think aloud while making their decisions. Think-aloud protocols also allowed for better understanding of the strategy development process during performance, and tested whether causal analysis leads to more effective strategies (Ericsson \& Simon, 1993; Schweiger, Anderson, \& Locke, 1985). In order to examine whether thinking aloud affected performance, we also included groups that did not produce thinkaloud protocols while they performed the decision-making task.

\section{Experiment 2 Method}

Participants and Design. Participants were undergraduates from the Georgia Institute of Technology. They were given extra credit for participating. They were randomly assigned to one of six experimental groups that were created by factorially combining causal model (Direct-Cause versus Common-Cause versus No-Model) and think-aloud (think-aloud protocol versus no think-aloud protocol). The resulting numbers of participants were: Common-Cause Think-aloud, $N=21$, Direct-Cause Think-aloud, $N=21$, No-Model Thinkaloud, $N=17$, Common-Cause No Think-aloud, $N=18$, Direct-Cause No Think-aloud, $N=$ 18, No-Model No Think-aloud, $N=14$.

Materials. Participants were presented a single business scenario consisting of a one-shot, non-iterative prisoner's dilemma-type task very similar to those used in the first experiment though no temporal information was provided. The text of the scenario follows:

"Imagine being the owner of a large winery in southern France. The year is almost done and you have to decide on your pricing strategy for next year. Your profit from sales depends on the price you set and on the price your main competitor sets. The following table shows your expected profits (number on the left side in each cell) and the expected profit of your competitor (number on the right in each cell).

\begin{tabular}{|l|l|l|}
\hline Profit in $\$$ & $\begin{array}{l}\text { Your competitor charges a } \\
\text { high price }\end{array}$ & $\begin{array}{l}\text { Your competitor charges a } \\
\text { low price }\end{array}$ \\
\hline You charge a high price & $\mathbf{\$ 1 0 0 , 0 0 0} / \$ 100,000$ & $\mathbf{\$ 6 0 , 0 0 0} / \$ 120,000$ \\
\hline You charge a low price & $\mathbf{\$ 1 2 0 , 0 0 0} / \$ 60,000$ & $\mathbf{\$ 8 0 , 0 0 0} / \$ 80,000$ \\
\hline
\end{tabular}


Both of you ended up having the same pricing strategy in 9 out of 10 years:You and your competitor charged high prices when the yield was good and medium prices when the yield was average."

This scenario was given to participants in the No-Model condition. For the DirectCause condition, a statement was added that read, "In the past, you based your prices almost every time on the yield of the wine. Your competitor waited for you to publish your price and then followed suit." In the Common-Cause condition, the statement read, "In the past, you and your main competitor based your prices almost every time on the quality of the wine." Hence, the Common-Cause scenario highlighted a common cause of both the participant's and the imagined opponent's outcomes in a situation, while the Direct-Cause scenario highlighted a direct cause between the participant's decision and the imagined opponent's choice.

Participants responded on a scale from 0 (absolutely charge a low price) to 100 (absolutely charge a high price). In this scenario, charging a low price is equivalent to defection and charging a high price is equivalent to cooperation. Because of the need to keep price and rating in the same direction, cooperation and defection were on ends opposite to those in Experiment 1.Consequently, for the results, this scale was reversed so that it was consistent in direction with the cooperation/defection scale (cooperation being closer to 0 and defection being closer to 100).

Procedure. Participants were tested individually and recorded with a lapel microphone linked directly to a computer for the think-aloud groups. The no think-aloud groups did exactly the same task except that they were not recorded or asked to verbalize their thoughts. Sessions lasted up to two hours, including subsequent exercises. Materials were presented on paper to allow participants the option of drawing/writing information as they studied the scenario. After participants completed a consent form, the scenario was presented, and the participant was instructed to read it out loud. Then participants were instructed to "think out loud" (Ericsson \& Simon, 1980). Think-aloud protocols have been shown to be an effective method that leaves thought processes relatively unchanged (Ericsson \& Simon, 1993). They were told to speak about whatever came to mind and, to avoid disrupting normal performance as much as possible (Berardi-Coletta et al., 1995), they were not directed to provide reasons for their thoughts or answers until after the task was completed (although everything they said was recorded). After reading and providing an answer for the scenario, participants were also asked to justify their response. For more information on the full procedure, see Robinson (2006).

\section{Experiment 2 Results}

Ratings. Mean ratings for each condition are shown in Table 2. A two-way ANOVA crossing think-aloud (yes or no) with model condition (Common-Cause, Direct-Cause, and 


\section{Table 2.}

Experiment 2: Mean Rating of Willingness to Defect as a Function of Causal Model and Think-aloud Protocol (standard errors in parentheses)

\begin{tabular}{|l|c|c|c|}
\cline { 2 - 4 } \multicolumn{1}{c|}{} & \multicolumn{3}{c|}{ Type of Causal Model } \\
\cline { 2 - 4 } \multicolumn{1}{c|}{} & Direct-Cause & Common-Cause & No-Model \\
\hline No Think-aloud & $59.7(8.3)$ & $78.7(6.2)$ & $67.1(6.6)$ \\
\hline Think-aloud & $36.4(7.9)$ & $42.4(5.9)$ & $59.7(7.1)$ \\
\hline Mean & $47.2(5.6)$ & $59.2(5.1)$ & $63.1(5.2)$ \\
\hline
\end{tabular}

No-Model) revealed a significant effect of think-aloud, $F(1,103)=14.82, p<0.001$. Participants were more likely to cooperate with think-aloud instructions $(M=45.3, S E=4.1)$ than without $(M=68.6, S E=4.2)$. Under think-aloud instructions, participants were being observed directly and perhaps were more likely therefore to want to impress the experimenter with their strategic skill or to avoid appearing selfish. No interaction between the two independent variables was observed, $F(2,103)=1.98$, n.s.

This analysis also revealed a trend for model type, $F(2,103)=2.69, p=0.073$. As our hypothesis concerned only the Direct-Cause versus Common-Cause comparison where we expected direct cause to be more cooperative (i.e., lower rating) than Common-Cause, we compared them directly and found the difference between them significant for no think-aloud participants, $t(34)=1.84$, one-tailed $p<0.05$, but not significant for think-aloud participants $(t<1){ }^{2}$

Qualitative Strategy Coding. Because rich think-aloud protocols were collected in Experiment 2, we were able to distinguish Direct-Cause strategies from other strategies and there were more categories of other strategies. This was not possible in Experiment 1 because people did not spontaneously report using a Direct-Cause strategy in their post hoc justifications (it was only possible to ascertain indications about the type of causal model assumed but not the strategy). A team of raters categorized responses of participants according to strategy type (see Appendix B for criteria used).Two raters coded each participant, coming to consensus if there were disagreements. A variety of strategies was reported (Figure 2).

Three of the most popular were (across all conditions): (a) Direct-Cause Strategy (20.4\%) - participant assumes he or she can impact opponent's behavior and gain a favorable outcome as a result, (b) Maximizing Payoff (18.4\%)_participant tries to get the highest payoff regardless of what opponent does, (c) Being Fair (14.3\%)_participant tries to follow concept of equitable distribution. Note that there was a sizable increase of a Direct-Cause strategy for participants in the Direct-Cause condition (although there were too few cases to do a meaningful statistical analysis). 
Figure 2. Percentages of Each Coded Strategy in Exp. 2

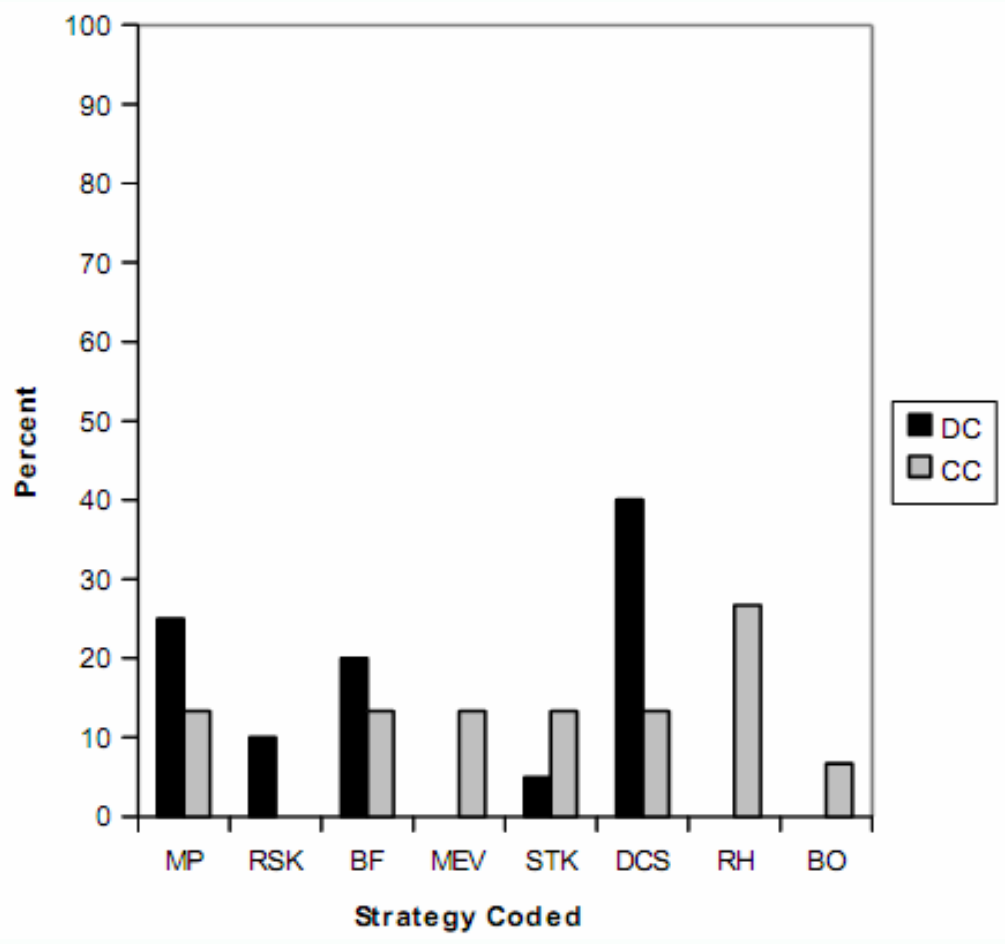

Note: $\mathrm{MP}=$ Maximum Payoff, RSK = Risk Aversion, BF = Being Fair, MEV = Maximum Expected Value, STK = Stackelberg Heuristic, DCS = Direct-Cause Strategy, RH = Red Herring, BO = Beating Opponent. DC = DirectCause Condition, $\mathrm{CC}=$ Common-Cause Condition.

Our primary hypothesis was that individuals using a Direct-Cause strategy would tend to choose the non-dominant option (i.e., give lower ratings). Consequently, the mean level of ratings was analyzed conditioned on whether a participant was categorized as using a Direct-Cause strategy or not. There were large differences in level of rating dependent upon whether someone chose a Direct-Cause strategy $(M=21.50, S E=4.28)$ or not $(M=$ $50.10, S E=4.57$, for the other strategies combined). In spite of the small sample sizes, the difference was significant, $t(57)=-2.76, p<0.05$. Irrespective of the condition a person was in, if he or she chose to infer a direct cause relation with an opponent, then he or she was more likely to cooperate.

Discussion. Despite the limited sample size, the patterns of data support the notion that causal models can influence choice in a competitive business context. Specifically, Direct-Cause instructions can lead to higher rates of cooperation than Common-Cause or No-Model instructions. This was particularly clear when focusing on the difference between causal instructions in the no think-aloud groups. Furthermore, an assumption of a particular causal model can influence choices even when no causal information is given. 
Second, the strategy a participant uses, regardless of experimental condition, can indicate whether that person will choose the dominant or non-dominant option in this type of task. Finally, people were more cooperative when talking aloud during choice.

\section{General Discussion}

Several key findings emerged from the current experiments. Individuals who were given or assumed a Direct-Cause model tended to be more cooperative when confronted with an economic decision-making problem. This tendency was found by comparing groups and also within the same participant from one scenario to another.Furthermore, temporal cues were not enough for an individual to infer a particular causal relationship. A delay in the sequence of decisions did not imply a direct cause between the participant and the opponent's decision. Finally, a variety of strategies were used to solve these problems, and the strategies that were indicative of a direct cause led to more cooperation.Causal model sensitivity and strategy choice influence whether a person will cooperate or not on an economic/financial prisoner's dilemma-like problem. If a person believes he or she has the ability to affect the opponent's behavior, then that individual is more likely to cooperate. These findings converge with other demonstrations that the causal model people impose on a situation influences their choice (Hagmayer \& Sloman, 2009; Sloman 2005).

Participants were given the same expected value information and probabilities across all problems, so utility theory alone cannot explain the shift in preference in the presence of different causal structures. In all problems, participants were told that $90 \%$ of the time their choice was the same as the opponent's. But the underlying cause of this probability was different depending on the causal model instructions. This causal information was enough to lead to higher rates of cooperation when participants were told that the percentage was due to a direct effect on the opponent's behavior instead of by chance events based on a common cause.

Are temporal cues nothing more than a proxy for causal inferences, and will they override the causal model presented? From the data in Experiment 1, the answers to both parts of this question are negative. There was no difference between the two temporal conditions. No differences for Common-Cause items were predicted under the assumption that causal information would trump temporal information. If told a common cause affected the participant's and the opponent's decisions (which were independent), then regardless of the timing of the choice, the participant was more likely to choose the dominant option. This is a reasonable strategy because participants would not have a basis to assume the opponent's decision will match and be affected by their decision.

In the absence of causal information, it was predicted that participants would assume a Direct-Cause relationship when their decision preceded the opponent's decision (sequential) or a Common-Cause relationship when the decisions were made simultaneously.This prediction was made under the assumption that temporal cues are proxies for 
causal information (e.g., Morris et al., 1998). ${ }^{3}$ Apparently, temporal cues were not treated like causal information as no difference in choice emerged when the temporal relations were simultaneous versus delayed. People did not assume a delay indicates a direct cause (i.e., that they could directly affect the opponent's choice). This contrasts directly with findings of Morris et al. (1998). The difference may have to do with the presence of explicit causal structure in other conditions in the current experiments but not in Morris et al.Participants may have not inferred causal from temporal structure here because they believed that they would be told of any causal structure they were expected to use.

How does causal information affect strategies in decision making? In both experiments, a wide variety of strategies was observed. In the first experiment, the distributions of strategies shifted from one causal model to the next. That is, on Common-Cause and No-Model items, participants had a higher percentage of strategies that focused on maximizing profit regardless of the opponent's choice or on beating the opponent (leading to more choices of the dominant option). In contrast, for Direct-Cause items, strategies concerned directly affecting the opponent (leading to higher levels of cooperation). This supports Moore's (1994) assertion that individuals will switch strategies based on what the problem affords.

One possible interpretation of these data is that the introduction of a Direct-Cause structure produced an asymmetry in social roles. Perhaps the first player was perceived as more of a leader whose action should be honored, therefore making the second player more likely to cooperate. A similar idea is discussed in the literature on game theory as the first mover advantage (cf. Dixit \& Skeath, 1999). Given that there is a Direct-Cause relation, the first player has the opportunity to cause the second player to pick an option that is advantageous for the first player. However, we saw no evidence of either course of reasoning in the strategy reports or think-aloud protocols.

Another key finding was that individuals coded as using a strategy consistent with a direct cause were much more likely to cooperate and individuals coded as focusing on dominance and payoffs were more likely to defect.Thus, although participants may have not directly thought about the causal underpinnings of the problem, its causal basis did shape the way they thought while choosing an option.

\section{Conclusion}

What can we say about the determinants of cooperation in two-player Prisoner's Dilemma type problems in an economic/financial context? Our first observation is that people use a variety of strategies and so we expect that cooperation has multiple determinants for different people and even for the same person on different occasions. Do people cooperate out of moral principle? We did find that "being fair" was one of the more common strategies. Nevertheless, it was still relatively rare and much less frequent than the incidence of cooperation. This suggests that not all cooperation is the result of moral principle.

- volume 3, no. 1 (Fall 2010) 
A second possible explanation is that cooperation is a self-presentation effect; people want to appear cooperative in front of an experimenter. Although our strategy reports showed no evidence for this, the difference we observed between think-aloud protocol and no think-aloud protocol participants in Experiment 2 did. People were more likely to cooperate if they thought aloud, suggesting either that verbalizing thoughts changes the thought process to in some way increase cooperation (which has been argued against by Ericsson \& Simon, 1993) or that people are more likely to cooperate if they know their thought process is being inspected by a third party.

Third, people might cooperate in order to prove to themselves that they have positive attributes related to being cooperative. We found no evidence for this account although that might be because our studies were not designed to provide such evidence.

Our final two accounts involve causal reasoning. First, people could cooperate because of backward causal reasoning, that cooperation will cause one to have positive personality characteristics, or to make the world a cooperative place. We did not see any evidence for backward causal reasoning. Second, they could cooperate because of the imposition of causal structure, the belief that one's action will affect one's opponent whether it will or not. We did observe evidence consistent with this hypothesis: people were sensitive to causal structure in the sense that their choices change when causal structure does, people frequently reported that their action would influence their opponent even when no model was given, and they sometimes reported strategies consistent with beliefs of a direct cause even in the No-Model condition.

Given the economic/financial context of the scenarios, causal structure can have important implications for understanding the ways individuals make financial decisions (and their beliefs of control). For example, an entrepreneur deciding on a marketing strategy may make decisions based on a faulty belief in affecting his or her competitor's strategies. Or, an investor may use a causal model to estimate (or in many common cases, overestimate) the amount of influence she or he may have on a particular sector of interest. If an investor is accurate in how much direct influence he or she has on a sector, then choosing to invest a greater amount may bring in more buyers to contribute funding, which will benefit everyone involved. Of course, this assumes that there is a reasonably high chance of others following suit, which may not be unreasonable in certain cases (e.g., when Warren Buffet invests in a small sector, or George Soros focuses on buying a specific currency, chances are others will join, which can positively affect the sector's success).This is an example of utilizing a Direct-Cause model and choosing to cooperate (i.e., invest a larger amount) in the hopes that others will follow and do the same.

In sum, people are both moral and causal reasoners in ways not captured by rational models of choice. Causal model theory (Nozick, 1969; Pearl, 2000; Sloman, 2005) provides a better basis for understanding human reasoning in economic cooperation problems. 


\section{Endnotes}

1. Another possibility would have been to limit analyses to participants who selected the same strategy on both items within a set, but that would have eliminated a sizable number of participants (50-70\%, depending on the type of problem). The distribution of strategies was similar (if only focusing on the major strategies), and the analyses of choice based on strategy within a block were consistent.

2. Experiment 2 showed a pattern similar to that of Experiment 1 although Experiment 1 had greater power to detect differences. In order to detect a medium effect size with a reasonable amount of power (say an $80 \%$ chance that a difference of this size would be significant), an $\mathrm{N}$ of 84 would have been necessary in a between-subjects study of this type (and logistically unfeasible for a qualitative study). The present sample size barely yielded power of 0.64 , based on a power analysis

3. An alternative conception is that the order of plays affects which equilibria the second player considers (Muller \& Sadanad, 2003; Weber, Camerer, \& Knez, 2004).

\section{References}

Berardi-Coletta, B., Buyer, L., Dominowski, R., \& Rellinger, E. (1995). Metacognition and problem-solving: A process-oriented approach. Journal of Experimental Psychology: Learning, Memory, and Cognition, 21(1), 205-223.

Camerer, C.F.(1997).Progress in behavioral game theory.Journal of Economic Perspectives, 11(4), 167-188.

Colman, A. (2003). Cooperation, psychological game theory, and limitations of rationality in social interaction. Behavioral and Brain Sciences, 26, 139-198.

Colman, A., \& Bacharach, M. (1997). Payoff dominance and the Stackelberg heuristic. Theory and Decision, 43, 1-19.

Davis, L. H. (1985). Prisoners, paradox, and rationality. In R. Campbell \& L. Sowden (Eds.), Paradoxes of rationality and cooperation: Prisoner's dilemma and Newcomb's problem. Vancouver: University of British Columbia Press.

Dixit, A. K., \& Skeath, S. (1999). Games of strategy. New York:W.W. Norton \& Company.

Ericsson, A., \& Simon, H. (1980). Verbal reports as data. Psychological Review, 87(3), 215251.

Ericsson, A., \& Simon, H. (1993). Protocol analysis: Verbal reports as data. Cambridge, MA: MIT Press.

Goeree, J.K., \& Holt, C. A. (2001).Ten little treasures of game theory and ten intuitive contradictions. The American Economic Review, 91(5), 1402-1422.

Hagmayer,Y., \& Sloman, S.A.(2005). Causal models of decision-making:Choice as intervention. In B. G. Bara, L. Barsalou, \& M. Bucciarelli (Eds.), Proceedings of the Twenty-Seventh Annual Conference of the Cognitive Science Society.

- volume 3, no. 1 (Fall 2010) 
Hagmayer, Y., \& Sloman, S. A. (2009). Decision makers conceive of their choice as intervention. Journal of Experimental Psychology: General, 138, 22-38.

Hedden, T., \& Zhang, J. (2002). What do you think I think you think? Strategic reasoning in matrix games. Cognition, 85, 1-36.

Langer, E. J. (1975). The illusion of control. Journal of Personality and Social Psychology, 32, 311-328.

Langer, E. J., \& Roth, J. (1975). Heads I win, tails it's chance: The illusion of control as a function of the sequence of outcomes in a purely chance task. Journal of Personality and Social Psychology, 32, 951-955.

Luce, R. D., \& Raiffa, H. (1957). Games and decisions. New York: John Wiley and Sons.

Markoczy, L. (2004). Multiple motives behind single acts of cooperation. The International Journal of Human Resource Management, 15(6), 1018-1039.

Moore, F. C. T. (1994). Taking the sting out of the prisoner's dilemma. The Philosophical Quarterly, 44(175), 223-233.

Morris, M.W., Sim, D. L. H., \& Girotto, V. (1998). Distinguishing sources of cooperation in the one-round prisoner's dilemma:Evidence for cooperative decisions based on the illusion of control. Journal of Experimental Social Psychology, 34, 494-512.

Muller, R.A., \& Sadanad,A. (2003). Order of play, forward induction, and presentation effects in two-person games. Experimental Economics, 6, 5-25.

von Neumann, J., \& Morgenstern, O. (1953). Theory of games and economic behavior. 3rd ed. Princeton, NJ: Princeton University Press.

Nisbett, R.E., \&Wilson,T.D.(1977).Telling more than we can know:Verbal reports on mental processes. Psychological Review, 84(3), 231-259.

Nozick, R. (1969). Newcomb's problem and two principles of choice. In N. Rescher (Ed.), Essays in honor of Carl G. Hempel.Dordrecht, Netherlands: D. Reidel.

Nozick, R. (1993). The nature of rationality. Princeton, NJ: Princeton University Press.

Pearl, J.(2000). Causality: Models, reasoning, and inference. Cambridge: Cambridge University Press.

Robinson, A. E. (2006). The impact of causality, strategies, and temporal cues on games of decision. Unpublished doctoral dissertation, Georgia Institute of Technology.

Schweiger, D.M., Anderson, C. R., \& Locke, E. A. (1985).Complex decision making:A longitudinal study of process and performance. Organizational Behavior and Human Decision Processes, 36, 245-272.

Shafir, E., \& Tversky, A. (1992).Thinking through uncertainty: Nonconsequential reasoning and choice. Cognitive Psychology, 24(4), 449-474.

Sloman, S. A. (2005). Causal models: How people think about the world and its alternatives. New York: Oxford University Press.

Sloman, S. A., \& Hagmayer, Y. (2006). The causal psycho-logic of choice. Trends in Cognitive Sciences, 10, 407-412. 
Stahl, D. O., \& Wilson, P.W. (1995). On players' models of other players: Theory and experimental evidence. Games and Economic Behavior, 10, 218-254.

Weber, R. A., Camerer, C.F., \& Knez, M. (2004).Timing and virtual observability in ultimatum bargaining and "weak link" coordination games. Experimental Economics, 7, 25-48.

\section{Appendix A}

Experiment 1.Sample Scenario 1 (Common-Cause, Simultaneous)

Imagine you are a senior official at the Bank of Japan. You are interested in establishing a position containing a group of currencies. It is necessary to decide whether to buy dollars (\$) or euros $(€)$ and you want to choose the action that generates the most profit for you.

Your main competitor is another large federal bank.Your expected profit from these contracts is dependent upon a combination of whether you decide to buy dollars (\$) or euros $(€)$ and whether your competitor decides to buy dollars (\$) or euros $(€)$.

In the past, you chose whether or not to buy dollars $(\$)$ or euros $(€)$ and your competitor had to make his or her decision independently. Both you and your competitor make your decisions without knowledge of the other's decision. By chance, $90 \%$ of the time you both have chosen to buy the same currency in the past. You both independently base your choices on economic data that can affect the value of these currencies.

Regular market access results in you choosing which to buy at exactly the same time your competitor makes the choice. Consequently, your competitor's choice will be unknown at the time of your decision.

The table below shows your expected profits (bold number on the left side in each cell) and the expected profit of your competitor (number on the right in each cell).

\begin{tabular}{|l|l|l|}
\cline { 2 - 3 } \multicolumn{1}{c|}{} & Your competitor buys dollars $(\$)$ & Your competitor buys euros $(€)$ \\
\hline You buy dollars $(\$)$ & $\mathbf{\$ 1}$ billion / \$1 billion & $\mathbf{\$ 5 0}$ million / \$1.2 billion \\
\hline You buy euros $(€)$ & $\mathbf{\$ 1 . 2}$ billion / \$50 million & $\mathbf{\$ 1 0 0}$ million / \$100 million \\
\hline
\end{tabular}

Experiment 1.Sample Scenario 2 (Common-Cause, Sequential)

Imagine you are in charge of marketing for a major supermarket chain.You are interested in launching an intensive marketing campaign. It is necessary to decide whether to market the produce section or the meat section and you want to choose the action that generates the most profit for you.

Your main competitor is another well-known supermarket chain. Your expected profit from these marketing campaigns is dependent upon a combination of whether you decide to market produce or meat and whether your competitor decides to market produce or meat.

- volume 3, no. 1 (Fall 2010) 
In the past, you chose the product to market and your competitor had to make his or her decision independently. Both you and your competitor make your decisions without knowledge of the other's decision. By chance, $90 \%$ of the time you both have chosen to market the same items in the past. You both independently base your choices on survey data from potential customers that can impact the profitability of each campaign.

The regular timeline of your company results in you choosing whether to market produce or meat first, and then your competitor will choose second. Consequently, your competitor's choice will be unknown at the time of your decision.

The table below shows your expected profits (bold number on the left side in each cell) and the expected profit of your competitor (number on the right in each cell).

\begin{tabular}{|l|l|l|}
\cline { 2 - 3 } \multicolumn{1}{c|}{} & Your competitor markets produce & Your competitor markets meat \\
\hline You market produce & $\mathbf{\$ 1 0 0 , 0 0 0} / \$ 100,000$ & $\mathbf{\$ 6 0 0 , 0 0 0} / \$ 50,000$ \\
\hline You market meat & $\mathbf{\$ 5 0 , 0 0 0} / \$ 600,000$ & $\mathbf{\$ 5 0 0 , 0 0 0} / \$ 500,000$ \\
\hline
\end{tabular}

\section{Experiment 1.Sample Scenario 3 (Direct-Cause)}

Imagine you are a real estate investor in San Francisco and are interested in buying a particular set of properties. However, several other investors are also bidding on the same set of properties. It is necessary to decide whether to make a high or a low bid. You want to choose the action that will generate the most profit for you.

Your main competitor is another well-known real estate investor in the same area. Your subsequent expected profit from the set of properties is dependent upon a combination of whether you decide to bid high or low and whether your competitor decides to bid high or low.

In the past, you chose the bid level and your competitor waited for you to announce your choice, then made his or her decision based upon yours. Consequently, you both end up doing the same thing most times-in fact, $90 \%$ of the time he or she bid high after you bid high, and he or she bid low after you bid low. Your decision is based on property value information that can affect the value of this set of properties.

The regular bidding system results in you entering a bid first, and then your competitor will enter a bid second. Consequently, your competitor's bid level will be unknown at the time of your decision.

The table below shows your expected profits (bold number on the left side in each cell) and the expected profit of your competitor (number on the right in each cell).

\begin{tabular}{|l|l|l|}
\cline { 2 - 3 } \multicolumn{1}{c|}{} & Your competitor bids high & Your competitor bids low \\
\hline You bid high & $\mathbf{\$ 5 0 0 , 0 0 0} / \$ 500,000$ & $\mathbf{\$ 4}$ million $/$ \$100,000 \\
\hline You bid low & $\mathbf{\$ 1 0 0 , 0 0 0} / \mathbf{\$ 4}$ million & $\mathbf{\$ 3 . 5}$ million $/$ \$3.5 million \\
\hline
\end{tabular}


Experiment 1.Sample Scenario 4 (Direct-Cause)

Imagine you are the Vice President of Research and Development at Googleplex and you are interested in developing a new tool for customers. Due to budget constraints, you can only choose one tool to develop. It is necessary to decide whether to develop a new mapping function or a new desktop search assistant. You want to choose the tool that generates the most sales for you.

Your main competitor is another well-known technology company. Your expected sales from these products are dependent upon a combination of whether you decide to develop the mapping function or desktop search assistant and whether your competitor decides to develop the mapping function or desktop search assistant.

In the past, you chose which product to focus the research budget on and your competitor waited for you to announce your choice, then made his or her decision based upon yours. Consequently, you both end up doing the same thing most times-in fact, $90 \%$ of the time he or she chose to develop the same product after your choice. Your decision is based on market constraints that determine the most opportunity for sales volume.

The regular company timeline results in you choosing which product to invest the research budget in first, and then your competitor will choose second. Consequently, your competitor's choice will be unknown at the time of your decision.

The table below shows your expected sales (bold number on the left side in each cell) and the expected sales of your competitor (number on the right in each cell).

\begin{tabular}{|l|l|l|}
\cline { 2 - 3 } \multicolumn{1}{c|}{} & $\begin{array}{l}\text { Your competitor develops } \\
\text { a mapping function }\end{array}$ & $\begin{array}{l}\text { Your competitor develops } \\
\text { a desktop search assistant }\end{array}$ \\
\hline You develop mapping function & $\mathbf{3 0 0 , 0 0 0 / 3 0 0 , 0 0 0}$ & $\mathbf{5 0 , 0 0 0} / 360,000$ \\
\hline You develop desktop search assistant & $\mathbf{3 6 0 , 0 0 0 / 5 0 , 0 0 0}$ & $\mathbf{7 5 , 0 0 0} / 75,000$ \\
\hline
\end{tabular}

Experiment 1. Sample Scenario 5 (No-Model, Simultaneous)

Imagine you are a politician running for state senate. You are interested in funding a large media campaign for the election. It is necessary to decide whether to fund a television or radio/internet campaign and you want to choose the action that generates the highest poll percentage numbers for you.

Your main competitor is another politician running for the same senate seat. Your expected poll percentage numbers from these media campaigns are dependent upon a combination of whether you decide to use television or radio/internet and whether your competitor decides to use television or radio/internet.

In the past, you and your competitor have chosen the same type of media campaign $90 \%$ of the time. Your decision is based on survey data showing the most effective way to reach/influence the most people.

Regular contract requirements of the different media results in you choosing whether

- volume 3, no. 1 (Fall 2010) 
to use television or radio/internet at exactly the same time your competitor makes the choice. Consequently, your competitor's choice will be unknown at the time of your decision.

The table below shows your expected poll percentages (bold number on the left side in each cell) and the expected poll percentages of your competitor (number on the right in each cell).

\begin{tabular}{|c|c|c|}
\hline & Your competitor uses television & $\begin{array}{l}\text { Your competitor uses } \\
\text { radio/internet }\end{array}$ \\
\hline You use television & $20 \% / 20 \%$ & $60 \% / 10 \%$ \\
\hline You use radio/internet & $10 \% / 60 \%$ & $\mathbf{5 0 \% / 5 0 \%}$ \\
\hline
\end{tabular}

Experiment 1.Sample Scenario 6 (No-Model, Sequential)

Imagine you are the owner of a successful Cajun restaurant. You are interested in establishing a new restaurant in another city. It is necessary to decide whether to build it in Chicago or Miami and you want to choose the place that generates the most yearly profit for you.

Your main competitor is the owner of another well-known Cajun restaurant and is also looking to establish in these areas. Your expected profit from this new restaurant is dependent upon a combination of whether you choose Chicago or Miami and whether your competitor chooses Chicago or Miami.

In the past, you and your competitor have chosen to build in the same city $90 \%$ of the time. Your decision is based on survey market testing research that indicates the level of demand in each city.

Regular contractor demand results in you choosing to build in Chicago or Miami first, and then your competitor will choose second. Consequently, your competitor's choice will be unknown at the time of your decision.

The table below shows your expected yearly profit (bold number on the left side in each cell) and the expected yearly profit of your competitor (number on the right in each cell).

\begin{tabular}{|l|l|l|}
\cline { 2 - 3 } \multicolumn{1}{c|}{} & Your competitor chooses Chicago & Your competitor chooses Miami \\
\hline You choose Chicago & $\mathbf{\$ 1 . 2}$ million $/ \$ 1.2$ million & $\mathbf{\$ 1 0 0 , 0 0 0} / \$ 1.5$ million \\
\hline You choose Miami & $\mathbf{\$ 1 . 5}$ million $/ \$ 100,000$ & $\mathbf{\$ 2 5 0 , 0 0 0} / \$ 250,000$ \\
\hline
\end{tabular}

\section{Appendix B:Experiment 2 Coding Scheme}

1. Beat Your Opponent (BO):The strategy is simply to outsell or "beat" your opponent, regardless of earning the maximum payoff. 
2. Being Fair (BF): The participant closely follows the rules that are spelled out in the scenario, and uses these rules to make his or her decision. The participant does not want to take unfair advantage of his or her opponent. This strategy also includes following unspoken rules or traditions.

3. Direct-Cause Strategy (DCS):The participant chooses the option that will impact the opponent's behavior, allowing him or her to achieve the desirable outcome.

4. Intuition (I):The participant's choice is dictated by intuition or extraneous information.

5. Maximum Expected Value (MEV):The goal of the participant is to earn the highest payoff given a particular probability of occurrence. This differs from MP as it takes into account likelihood of success.

6. Maximum Payoff (MP): The goal of the participant is to earn the largest payoff (the preferable outcome), regardless of the opponent's actions or the likelihood of success.

7. Risk Aversion (RSK): The participant seeks a predictable outcome and, as a result, may be willing to settle for an outcome that is less desirable.

8. Reasoning by Analogy (RBA):The participant's choice is dictated by the belief that his or her opponent will act in a manner similar to his or her own given similar circumstances.

9. Red Herring (RH):The participant utilizes only one piece of information from the scenario to solve the problem, disregarding the rest.

10. Not Enough Info (NEI): The participant feels like there is not enough information given in the problem to make a decision. The participant usually chooses a 50 on the rating scale.

11. Risk Aversion (RA): The participant tries to avoid losses at all costs, taking a defensive/protective posture.

12. Stackelberg Heuristic (STK): The participant assumes that his or her opponent will guess what strategy to use; in turn, he or she chooses the best counter-strategy. In other words, the strategy that the participant ends up utilizing is the OPTIMAL response to his or her OPPONENT'S best counterstrategy.

13. Unable to Code (UC): Unable to decipher what the participant is doing.

\section{Examples of the Most Common Strategies}

\section{Direct-Cause Strategy}

Example 1:"Okay, so if you charge a high price, um, and your competitor charges a low price, which isn't very likely from what it said his strategy was in setting his price, you would make less. But if you charged a low price, while your competitor charges a high price, then you would get twice as much as he did. So it's kind of like a prisoner's dilemma-type thing. But since he's set in his price, um, it says he's setting his price based on yours in the past, 
it's pretty fair to assume he's going to do that again. So it seems like you should charge a higher price because if he charges a low price along with you, if you decide to do that, then you'll both make less than, not that you want him to make more money. But you'll make less than if you just went ahead and charged the higher price with him charging it too, so I think you should, um, $95 \%$, to set high price."

Example 2:"My competitor charges a high price, then I get the most profit. So that sounds good so far.So I might want to make him charge a high price, which would get me $\$ 100,000$, which doesn't sound bad. So yeah, I'm going to go with charge a high price."

\section{Maximum Payoff}

Example:"Let's see, I'm just trying to, okay, your competitor charges a high price, okay, so when your competitor charges a high price, your expected profits are the same. When your competitor charges a high price and when you charge a high price your expected profits are the same. When you charge a lower price, your expected profits are more than his. Okay, your competitor charges a low price and you charge a high price, your expected profits are less than his. Okay, which price will you charge? Um, zero being absolutely charge low prices, 100 being absolutely charge high prices. Um, I would say, uh, to maximize your profit, the expected profit. I think you would, uh, charge a price in between the two, so I would say 50 for that. So, uh, your competitor's profit would probably be about the same as yours. So l'd say $50 . "$

\section{Being Fair}

Example:" $U m$, since the yield is above average and it says you tend to charge higher prices when the yield is higher.Um, and whenever you charge 100 , your competitor usually charges 100, according to this chart. And, um, so you want it to be about the same as your competitor. Cause if they are too different then it would cause problems, I guess." 